\title{
The Active Sampling of Gaze-Shifts
}

\author{
Giuseppe Boccignone $^{1}$ and Mario Ferraro $^{2}$ \\ 1 Dipartimento di Scienze dell'Informazione Universitá di Milano \\ via Comelico 39/41, 20135 Milano, Italy \\ boccignone@dsi.unimi.it \\ 2 Dipartimento di Fisica Sperimentale, Universitá di Torino \\ via Pietro Giuria 1, 10125 Torino, Italy \\ ferraro@ph.unito.it
}

\begin{abstract}
The ability to predict, given an image or a video, where a human might fixate elements of a viewed scene has long been of interest in the vision community.

In this note we propose a different view of the gaze-shift mechanism as that of a motor system implementation of an active random sampling strategy that the Human Visual System has evolved in order to efficiently and effectively infer properties of the surrounding world. We show how it can be exploited to carry on an attentive analysis of dynamic scenes.
\end{abstract}

Keywords: active vision, visual attention, video analysis.

\section{Introduction}

Gaze shifts are eye movements that play an important role: the Human Visual System (HVS) achieves highest resolution in the fovea and the succession of rapid eye movements (saccades) compensates the loss of visual acuity in the periphery when looking at an object or a scene that spans more than several degrees in the observer's field of view. Thus, the brain directs saccades to actively reposition the center of gaze on circumscribed regions of interest, the so called "focus of attention" (FOA), to sample in detail the most relevant features of a scene, while spending only limited processing resources elsewhere. An average of three eye fixations per second generally occurs, intercalated by saccades, during which vision is suppressed. Frequent saccades, thus, avoid to build enduring and detailed models of the whole scene. Apparently evolution has achieved efficient eye movement strategies with minimal neural resources devoted to memory [13].

In order to take into account these issues, much research has been devoted specially in the fields of computational vision (see, for instance, 91022]).

Interestingly enough, one point that is not addressed by most models is the "noisy", idiosyncratic variation of the random exploration exhibited by different observers when viewing the same scene, or even by the same subject along different trials [15]. Such variations speak of the stochastic nature of scanpaths. Indeed, at the most general level one can assume any scanpath to be the result of a random walk performed to visually sample the environment under the constraints of both the physical information provided by the stimuli (saliency or 
conspicuity) and the internal state of the observer, shaped by cognitive (goals, task being involved) and emotional factors. Under this assumption, the very issue is how to model such "biased" random walk.

In a seminal paper [6, Brockmann and Geisel have shown that a visual system producing Lévy flights implements an efficient strategy of shifting gaze in a random visual environment than any strategy employing a typical scale in gaze shift magnitudes. Lévy flights provide a model of diffusion characterized by the occurrence of long jumps interleaved with local walk.

To fully exploit diffusion dynamics, in [3, a gaze-shift model (the Constrained Lévy Exploration, CLE) was proposed where the scanpath is guided by a Langevin equation,

$$
\frac{d \mathbf{x}}{d t}=-U(\mathbf{x})+\xi
$$

on a potential $U(\mathbf{x})$ modelled as a function of the saliency (landscape) and where the stochastic component $\xi$ represents random vector sampled from a Lévy distribution (refer to 3 for a detailed discussion, and to 1112 for application to robot vision relying on Stochastic Attention Selection mechanisms). The basic assumption was the "foraging metaphor", namely that Lévy-like diffusive property of scanpath behavior mirrors Lévy-like patterns of foraging behavior in many animal species [24]. In this perspective, the Lévy flight, as opposed, for instance, to Gaussian walk, is assumed to be essential for optimal search, where optimality is related to efficiency, that is the ratio of the number of sites visited to the total distance traversed by forager [24]. An example depicting the difference between Gaussian and Lévy walks is provided in Fig. 1, In [4] a new method, the Lévy Hybrid Monte Carlo (LHMC) algorithm was presented, in which gaze exploration is obtained as a sampling sequence generated via a dynamic Monte Carlo technique. Interestingly enough the method described in [3] can be recovered as a special case of [4] .
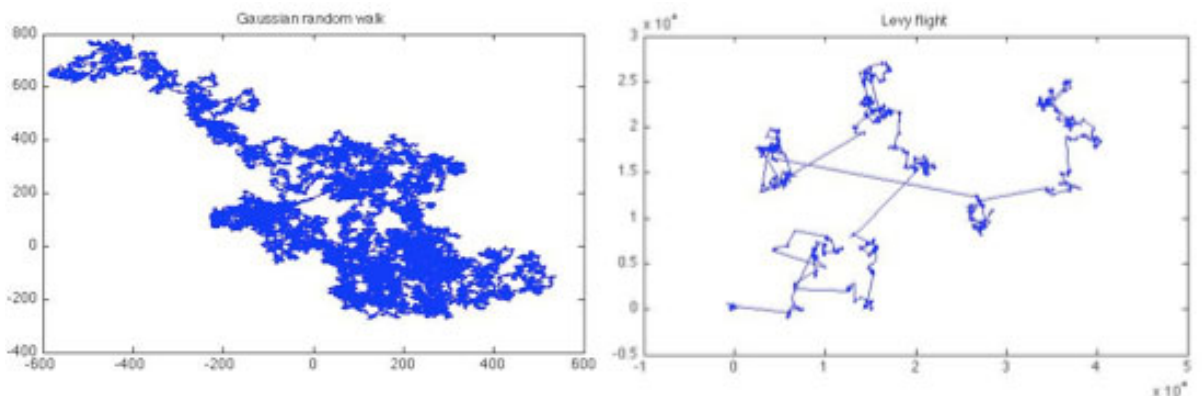

Fig. 1. Different walk patterns obtained through the method described in 7] (defined in the next section). Top: Gaussian walk (characteristic exponent or index of stability $\alpha=2)$; bottom: Lévy walk $(\alpha=1.2)$. Horizontal and vertical axes represent $(X, Y)$ coordinates; note the different scale at which the flight lenghts are obtained. 
This perspective suggested a different and intriguing view of the saccadic mechanism: that of a motor system implementation of an active random sampling strategy that the HVS has evolved in order to efficiently and effectively infer properties of the surrounding world. In this note we further develop this view, showing how it can be exploited to carry on an attentive analysis of dynamic scenes.

\section{Background}

The fact that a visual system producing Lévy flights implements an efficient strategy of shifting gaze in a visual environment should not be surprizing [6]3. Indeed, movements of some biological organisms can be represented as a trajectory constructed as a Random Walk Model (a simplified version of Eq. 1),

$$
\mathbf{x}_{t+1}=\mathbf{x}_{t}+\xi
$$

where $\xi$ is a random vector representing the displacement $\mathbf{x}_{t} \rightarrow \mathbf{x}_{t+1}$ occurring at time $t$. In other terms, a discrete-time continuous-space Lévy flight is a Markovian Random Walk process controlled by the conditional probability $P\left(\mathbf{x}_{t+1} \mid \mathbf{x}_{t}\right) d \mathbf{x}_{t+1} d \mathbf{x}_{t}$ for the walker to be in the region $\mathbf{x}_{t+1}+d \mathbf{x}_{t+1}$ at time $t+1$, if he was in the region $\mathbf{x}_{t}+d \mathbf{x}_{t}$ at time $t$. We restrict ourselves to

$$
P\left(\mathbf{x}_{t+1} \mid \mathbf{x}_{t}\right) \approx P\left(\mathbf{x}_{t+1}-\mathbf{x}_{t}\right)=P(\xi) \sim|\xi|^{-(1+\alpha)}
$$

for large $|\xi|$, namely the lenght of the single diplacement $\xi$. The nature of the walk is determined by the asymptotic properties of such conditional probability (see, for instance, 5]): for the characteristic exponent $\alpha \geq 2$ the usual random walk (brownian motion) occurs; if $\alpha<2$, the distribution of length jump is "broad" and the so called Lévy flights or Lévy walks take place 1 .

Intuitively, Lévy flights (walks) consist of many short flights and occasional long flights: sample trajectories of an object undergoing ordinary random walk and Lévy flights, are presented in Fig. 1.

Coming back to Eq. 2, one can simulate a Brownian walk (e.g. , $\alpha=2$ ) by sampling $\xi$ from a Normal distribution at each time step $t$; for what concerns Lévy motion, one could choose, for instance, to sample from a Cauchy distribution $(\alpha=1)$. However, this constrains to specific values of the characteristic exponent $\alpha$, which might become a limit for the analysis of natural phenomena. At the most general level we can choose $P(\xi)=f(\alpha, \beta, \gamma, \delta)$ to be an alpha-stable distribution [14] of characteristic exponent (index of stability), skewness, scale and location parameters $\alpha, \beta, \gamma, \delta$, respectively. In general there exists no simple closed form for the probability density function of stable distributions: they

\footnotetext{
${ }^{1}$ The Lévy Flight and the Lévy Walk are trajectories constructed in the same way spatially but differ with respect to time. The Lévy Flight displaces in space with one unit of time being spent for each element between turning points. It could be considered a discrete version of the Lévy Walk. The Lévy Walk on the other hand is a continuous trajectory and has a velocity vector.
} 
admit explicit representation of the density function only in the following cases: the Gaussian distribution $P(2,0, \gamma, \delta)$, the Cauchy distribution $P(1,0, \gamma, \delta)$ and the Lévy distribution $P(1 / 2,1, \gamma, \delta)$. Nevertheless, there are efficient numerical methods to generate samples $\xi \sim f(\alpha, \beta, \gamma, \delta)$ ([8], cfr. the Simulation Section).

Examples of Lévy flights and walks have been found in many instance of animal mouvement such as spider monkey [16], albatrosses [23], jackals 1] and in general such dynamics have been found to be be essential for optimal exploration in random searches [24].

It is also becoming apparent that other complex models can be subsumed within a Lévy flight paradigm [18. For example, in the composite Brownian walk (CBW), taking into account search patterns within a landscape of patchily distributed resources, a searcher moves in a straight-line between patches and adopts more localized Brownian movements within a patch. The search pattern is therefore adaptive because detection of a food item triggers switching from an extensive mode of searching for clusters of food to intensive within-cluster searching for individual prey items. Benhamou 2] showed that this CBW outperforms any Lévy-flight searching strategy with constant $\mu$. The CBW can, however, be interpreted as an adaptive Lévy-flight searching pattern in which the inter-patch straight-line motions correspond to Lévy flights with $\mu \rightarrow 1$ and where the intra-patch motion corresponds to a Lévy flight with $\mu=3$. This adaptive Lévy flight is an optimal strategy because $\mu \rightarrow 1$ is optimal for the location of randomly, sparsely distributed patches that once visited are depleted and because $\mu=3$ flights are optimal for the location of densely but random distributed within-patch resources [17. In complex environments a mixed strategy could be an advantage. For instance Reynolds and Frye [19, by analizing how fruit flies (Drosophila melanogaster) explore their landscape have reported that once the animals approach the odor, the freely roaming Lévy search strategy is abandoned in favour of a more localized (Brownian) flight pattern. Such mixed-type motion behavior is indeed the one taken into account in our active sampling strategy.

\section{The Active Sampling Strategy}

Denote $\mathbf{x}_{F O A}(t)=\mathbf{x}_{t}$ the oculomotor state which results, at time $t$, in a gaze fixation at a certain point of the world. The state of the world at time $t$ is then observed as "filtered" by the attentional focus set $\mathbf{x}_{t}$, and producing an observation likelihood $P\left(w_{t} \mid \mathbf{x}_{t}\right)$.

The observation likelihood is obtained as follows. Given a fixation point at time $t$ the current frame is blurred with respect to a Gaussian function centered at $\mathbf{x}_{t}$ in order to simulate foveation of the scene. Then, relying on motion features, a Bayesian surprise map $\mathcal{S} \mathcal{M}(t)=\left\{\mathcal{S M}(t)_{i}\right\}_{i=1}^{N}$ at each site $i \in \Im$, $\Im$ being the spatial support of current frame (see Itti and Baldi [9] for detailed discussion). Based on such map we sample a subset $\Im_{s}$ of salient interest points $\left\{\widetilde{\mathcal{S M}}(t)_{s}\right\}_{s=1}^{N s}$, where $N_{s}=\left|\Im_{s}\right|<N$, through a weighted random sampling: 
1. Sample:

$$
s \sim \operatorname{Unif}(\Omega)
$$

2. Sample:

$$
u_{s} \sim \operatorname{Unif}(0,1) .
$$

3. Accept, ${\widetilde{s_{t}}}^{s}$ with probability $P_{s}=\frac{\widetilde{\mathcal{S M}}(t)_{s}}{\mathcal{S \mathcal { M }}(t)_{\max }}$, if $u_{s}<P_{s}$.

Then, we subdivide the image into $L$ windows and let $P\left(w_{t}^{s}\right)$ be the probability that the $s$-th point falls in the window $w_{t}$ when $t \rightarrow \infty$, in other words $P\left(w_{t}^{s}\right)$ is the asymptotic probability distribution. Subregion partitioning of the image, which performs a coarse-graining of the saliency map, is justified by the fact that gaze-shift relevance is determined according to the clustering of salient points that occur in a certain region of the image, rather than by single points. Thus, the image is partitioned into $N w$ rectangular windows. For all $N s$ sampled points, each point occurring at site $s$, is assigned to the corresponding window, and probability $P\left(w_{t}=l \mid \mathbf{x}_{t}\right), l=1 \cdots N w$ is empirically estimated as

$$
P\left(w_{t}=l \mid \mathbf{x}_{t}\right) \simeq \frac{1}{N s} \sum_{k=1}^{N s} \chi_{k, l}
$$

where $\chi_{k, l}=1$ if $\widetilde{\mathcal{S M}}(t)_{k} \in w_{t}=l$ and 0 otherwise.

Under a sampled observation $w_{t} \sim P\left(w_{t}=l \mid \mathbf{x}_{t}\right)$, the observer has to determine the joint probability $P\left(\mathbf{x}_{t+1}, a_{t} \mid \mathbf{x}_{t}, w_{t}\right)$ of taking an action $a_{t}$ and of achieving the next state $\mathbf{x}_{t+1}$. We can rewrite such joint probability as:

$$
P\left(\mathbf{x}_{t+1}, a_{t} \mid \mathbf{x}_{t}, w_{t}\right)=P\left(\mathbf{x}_{t+1} \mid \mathbf{x}_{t}, a_{t}\right) P\left(a_{t} \mid \mathbf{x}_{t}, w_{t}\right) .
$$

The term $P\left(\mathbf{x}_{t+1} \mid \mathbf{x}_{t}, a_{t}\right)$ represents the dynamics, given action $a_{t}$, while $P\left(a_{t} \mid \mathbf{x}_{t}, w_{t}\right)$ is the probability of undertaking such action $a_{t}$ given the current state of affairs $\left(\mathbf{x}_{t}, w_{t}\right)$.

We can represent the action as the pair $a_{t}=\left(z_{t}, \theta_{t}\right)$, where $z_{t}$ is a discrete random variable $z_{t}=\left\{z_{t}=k\right\}_{k=1}^{K}, K$ being the number of possible actions, $\theta_{t}=\left\{\theta_{t}=k\right\}_{k=1}^{K}$ representer the parameters related to the action. Under the hypotheses motivated in the previous section, $z_{t}=\{1,2\}$, for foraging and exploratory behaviors, then

$$
P\left(a_{t} \mid \mathbf{x}_{t}, w_{t}\right)=P\left(z_{t}, \theta_{t} \mid \mathbf{x}_{t}, w_{t}\right)=P\left(z_{t} \mid \theta_{t}\right) P\left(\theta_{t} \mid a_{t}, b_{t}\right),
$$

where $a_{t}, b_{t}$ are the hyperparameters for the prior $P\left(\theta_{t} \mid a_{t}, b_{t}\right)$ on parameters $\theta_{t}$.

Since in our case the motor behavior is chosen among two possible kinds, $p(z=k \mid \theta)=\theta_{k}$ is a Binomial distribution whose conjugate prior is the Beta distribution, $p(\theta)=\operatorname{Beta}\left(\theta ; a_{t}, b_{t}\right)$.

Coming back to Eq[7, we write that oculomotor flight will be governed by a Binomial distribution,

$$
P\left(\mathbf{x}_{t+1} \mid \mathbf{x}_{t}, a_{t}\right)=\prod_{z_{t}}\left[P\left(\mathbf{x}_{t+1} \mid \mathbf{x}_{t}, \eta\right)\right]^{z_{t}},
$$


where $P\left(\mathbf{x}_{t+1} \mid \mathbf{x}_{t}, z_{t}=k\right)=P\left(\mathbf{x}_{t+1} \mid \mathbf{x}_{t}, \eta_{k}\right)$ is the flight generated according to motor behavior $z_{t}=k$ and regulated by parameters $\eta_{k}$. Here $P\left(\mathbf{x}_{t+1} \mid \mathbf{x}_{t}, \eta_{k}\right)$, is the probabilistic representation of the jump

$$
\mathbf{x}_{t+1}=\mathbf{x}_{t}+\xi_{k},
$$

where $\xi_{k} \sim f\left(\eta_{k}\right), f\left(\eta_{k}\right)=f\left(\alpha_{k}, \beta_{k}, \gamma_{k}, \delta_{k}\right)$ being an alpha-stable distribution of characteristic exponent, skewness, scale and location parameters $\alpha_{k}, \beta_{k}, \gamma_{k}, \delta_{k}$, respectively.

The last thing we have to take into account are the hyperparameters $a_{t}, b_{t}$ of the Beta distribution that govern the choice of motor behavior regime.

Following the discussion in the previous Section, here we assume that at each time $t$ these are "tuned" as a function of the order/disorder of the scene: intuitively, a completely ordered or disordered scenario will lead to longer flights so as to gather more information, whilst at the edge of order/disorder enough information can be gathered via localized exploration. This insight can be formalized as follows.

Consider again the probability distribution $P\left(w_{t}=l \mid \mathbf{x}_{t}\right)$. The corresponding Boltzmann-Gibbs-Shannon entropy is $S=-k_{B} \sum_{i=1}^{N} P\left(w_{t}=i \mid \mathbf{x}_{t}\right) \log P\left(w_{t}=\right.$ $\left.i \mid \mathbf{x}_{t}\right)$, where $k_{B}$ is the Boltzmann's constant; in the sequel, since dealing with images we set $k_{B}=1$. The supremum of $S$ is obviously $S_{\text {sup }}=\ln N$ and it is associated to a completely unconstrained process, that is a process where $S=$ const, since with reflecting boundary conditions the asymptotic distribution is uniform.

Following Shiner et al. [20] it is possible to define a disorder parameter $\Delta$ as $\Delta \equiv S / S_{\text {sup }}$ and an order parameter $\Omega$ as $\Omega=1-\Delta$ can be defined. Note that by virtue of our motion features and related Bayesian surprise, a disordered frame event will occur when either no motion (static scene) or extreme motion (many objects moving in the scene) is detected. Thus, the hyperparameter update can be written as

$$
\begin{gathered}
a_{(t)}=a_{(0)}+f_{a}\left(\Omega_{t}\right), \\
b_{(t)}=b_{(0)}+f_{b}\left(\Delta_{t}\right),
\end{gathered}
$$

and is simply computed as a counter of the events "ordered frame", "disordered frame", occurred up to time $t$, namely, $f_{a}\left(\Omega_{t}\right)=N_{\Omega_{t}}$ and $f_{b}\left(\Delta_{t}\right)=N_{\Delta_{t}}$.

\section{Simulation}

Simulation has been performed on the publicly available datasets, the BEHAVE Interactions Test Case (http://homepages.inf.ed.ac.uk/rbf/BEHAVE), the CAVIAR data set (http://homepages.inf.ed.ac.uk/rbf/CAVIAR/), the UCF Crowd Data set (http://server.cs.ucf.edu/vision/) The first comprises videos of people acting out various interactions, under varying illumination 


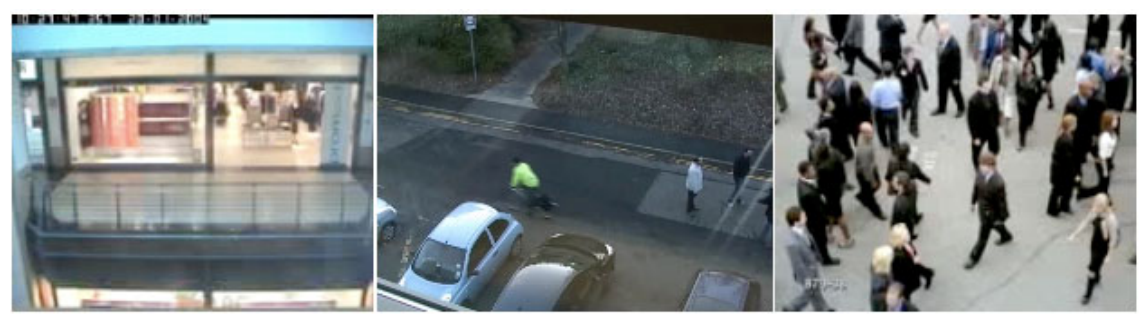

Fig. 2. Examples of videos used in the simulation. Left: video with no dynamic events happening from the CAVIAR data set; center: a video from the BEHAVE test set showing a few significative and temporally ordered dynamic events (one man cycling across the scene followed by a group of people interacting); right: a crowded scene from the UCF dataset.

conditions and spurious reflections due to camera fixed behind a window; the second include people walking alone, meeting with others, window shopping, entering and exiting shops; the third contains videos of crowds and other high density moving objects. The rationale behind the choice of these data sets stems from the possibility of dealing with scenes with a number of moving objects at a different level of complexity (in terms of order/disorder parameters as defined in the previous Section). At a glance, three representative examples are provided in Fig. 2,

Given a fixation point $\mathbf{x}_{t}$ at time $t$ (the frame center is chosen for $t=1$ ), the current RGB frame of the input sequence is blurred with respect to a Gaussian function centered at $x_{t}$ and down-sampled via a 3-level Gaussian pyramid. At the pre-attentive stage, optical flow features $\mathbf{v}_{n, t}$ are estimated on the lowest level of the pyramid following [21].

The related motion energy map is the input to the Bayesian surprise step, where the surprise map $\mathcal{S M}(t)$ is computed [9]. Then, through weighted random sampling, the set of salient interest points $\left\{\widetilde{\mathcal{S M}}(t)_{s}\right\}_{s=1}^{N s}$ is generated and $P\left(w_{t}=\right.$ $\left.l \mid \mathbf{x}_{t}\right)$ is estimated; for this purpose $N s=50$ interest points are sampled and we use $N w=16$ windows/bins, their size depending on the frame size $|\Im|$, to compute $\mathrm{Eq} 6$.

At this point we can compute the order/disorder parameters, and in turn the hyperparameters of the Beta distribution are updated via Eq. 11. This is sufficient to set the bias of the "behavioral coin" (Eq 8) and the coin is tossed (Eq. 91). This allows to choose parameters $\alpha_{k}, \beta_{k}, \gamma_{k}, \delta_{k}$ and to sample the flight vector $\xi$. For the examples shown here the Brownian regime is characterized by $\alpha_{1}=2, \beta_{1}=0, \gamma_{1}=|F O A|, \delta_{1}=0$ and the Lévy one by $\alpha_{2}=1.3, \beta_{2}=$ $0, \gamma_{2}=|F O A|, \delta_{2}=0$. Here $|F O A|$ indicates approximately the radius of a FOA, $|F O A| \approx 1 / 8 \min [w, h], w, h$ being the horizontal and vertical dimensions of the frame. 


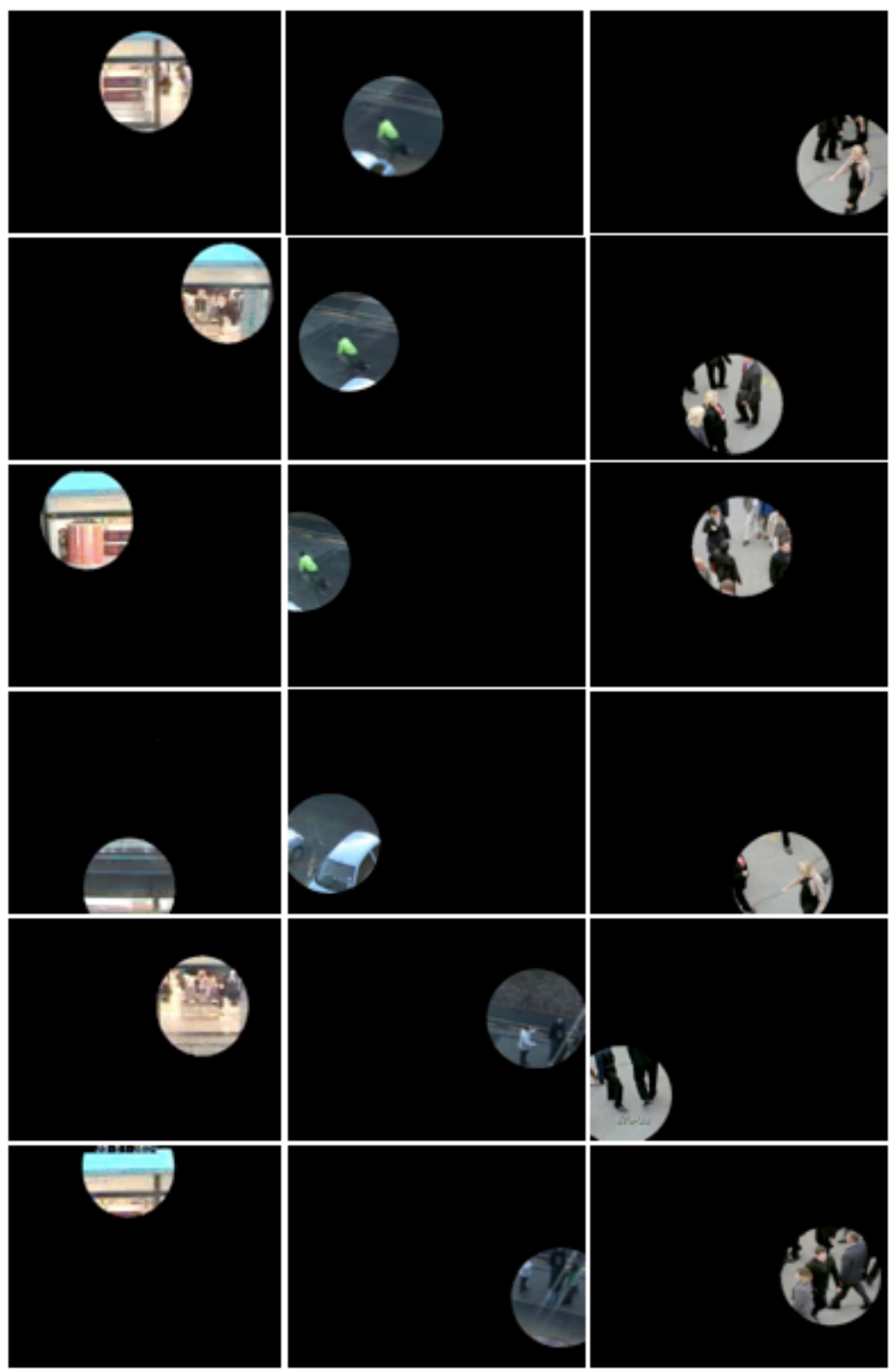

Fig. 3. An excerpt of typical results obtained along the simulation. Left and right columns shows that gaze samples the scene by principally resorting to a Lévy strategy as a result of no interesting dynamic events or too many dynamic events occurring in the scene. In the middle column a complex behavior is generated: a Brownian regime is maintained during an interesting event (resulting in bicyclist tracking), followed by a Lévy exploration to search for a new interesting event, ended by backing to a Brownian dynamics for sampling the new event of interest (people discussing). 
Given the parameters, an alpha-stable random vector $\xi$ can be sampled in several ways $[8]$. The one applied here [7] is the following.

1. Generate a value $Z$ from a standard stable $f(\alpha, \beta, 0,1)$ :

$$
\begin{gathered}
V \sim U\left[-\frac{\pi}{2}, \frac{\pi}{2}\right] ; \\
W \sim \exp (1) .
\end{gathered}
$$

2. If $\alpha \neq 1$ :

$$
Z=S_{\alpha, \beta} \frac{\sin \left(\alpha\left(V+B_{\alpha, \beta}\right)\right)}{\cos (V)^{1 / \alpha}}\left(\frac{\cos \left(V-\alpha\left(V+B_{\alpha, \beta}\right)\right)}{W}\right)^{\frac{1-\alpha}{\alpha}},
$$

where $S_{\alpha, \beta}=\frac{\arctan (\beta \tan (\pi \alpha / 2))}{\alpha}$ and $B_{\alpha, \beta}=\left(1+\beta^{2} \tan ^{2}(\pi \alpha / 2)\right)^{1 / 2 \alpha}$.

3 . If $\alpha=1$, then

$$
Z=\frac{2}{\pi}\left[\left(\frac{\pi}{2}+\beta V\right) \tan (V)-\beta \log \left(\frac{W \cos (V)}{\frac{\pi}{2}+\beta V}\right)\right] .
$$

Once a value $Z$ from a standard stable $f(\alpha, \beta, 0 ; 1)$ has been simulated, in order to obtain a value $\xi$ from a stable distribution with scale parameter $\gamma$ and location parameter $\delta$, the following transformation is required: $\xi=Z+\delta$ if $\alpha \neq 1$; $\xi=\gamma Z+\frac{2}{\pi} \beta \gamma \log (\gamma)+\delta$, if $\alpha=1$.

Eventually, the new FOA $\mathbf{x}_{t+1}$ is determined via Eq. 2.

An illustrative example, which is representative of results achieved on such data-set, is provided in Fig. 3, where the change of motor behavior regime is readily apparent as a function of the complexity of scene dynamics.

The system is currently implemented in plain MATLAB code, with no specific optimizations and running on a $2 \mathrm{GHz}$ Intel Core Duo processor, 2 GB RAM, under Mac OS X 10.5.8. As regards actual performance, most of the execution time is spent to compute the saliency map, which takes an average elapsed time of 0.8 secs per frame, whilst only 0.1 sec per frame is devoted to the FOA sampling. Clearly, the speed-up in this phase is due to the fact that once the set of salient interest points $\left\{\widehat{\mathcal{S M}}(t)_{s}\right\}_{s=1}^{N s}$ has been sampled, then subsequent computations only deal with $N s=50 \mid$ points, a rather sparse representation of the original frame.

\section{References}

1. Atkinson, R.P.D., Rhodes, C.J., Macdonald, D.W., Anderson, R.M.: Scale-free dynamics in the movement patterns of jackals. Oikos 98(1), 134-140 (2002)

2. Benhamou, S.: How many animals really do the Lévy walk? Ecology 88(8), 1962 1969 (2007)

3. Boccignone, G., Ferraro, M.: Modelling gaze shift as a constrained random walk. Physica A: Statistical Mechanics and its Applications 331(1-2), 207-218 (2004)

4. Boccignone, G., Ferraro, M.: Gaze shifts as dynamical random sampling. In: Proceedings of 2nd European Workshop on Visual Information Processing (EUVIP 2010), pp. 29-34. IEEE Press, Los Alamitos (2010) 
5. Bouchaud, J., Georges, A.: Anomalous diffusion in disordered media: statistical mechanisms, models and physical applications. Physics Reports 195, 127-293 (1990)

6. Brockmann, D., Geisel, T.: The ecology of gaze shifts. Neurocomputing 32(1), 643650 (2000)

7. Chambers, J.M., Mallows, C.L., Stuck, B.W.: A method for simulating stable random variables. J. Am. Stat. Ass. 71(354), 340-344 (1976)

8. Fulger, D., Scalas, E., Germano, G.: Monte Carlo simulation of uncoupled continuous-time random walks yielding a stochastic solution of the space-time fractional diffusion equation. Physical Review E 77(2), 21122 (2008)

9. Itti, L., Baldi, P.: Bayesian surprise attracts human attention. Vision Research 49(10), 1295-1306 (2009)

10. Itti, L., Koch, C., Niebur, E.: A model of saliency-based visual attention for rapid scene analysis. IEEE Trans. Pattern Anal. Machine Intell. 20, 1254-1259 (1998)

11. Martinez, H., Lungarella, M., Pfeifer, R.: Stochastic Extension to the AttentionSelection System for the iCub. University of Zurich, Tech. Rep (2008)

12. Nagai, Y.: From bottom-up visual attention to robot action learning. In: Proceedings of 8 IEEE International Conference on Development and Learning, IEEE Press, Los Alamitos (2009)

13. Najemnik, J., Geisler, W.S.: Optimal eye movement strategies in visual search. Nature 434(7031), 387-391 (2005)

14. Nolan, J.P.: Stable Distributions - Models for Heavy Tailed Data. Birkhauser, Boston (2011)

15. Privitera, C.M., Stark, L.W.: Algorithms for defining visual regions-of-interest: Comparison with eye fixations. IEEE Trans. Pattern Anal. Machine Intell. 22(9), 970-982 (2000)

16. Ramos-Fernandez, G., Mateos, J.L., Miramontes, O., Cocho, G., Larralde, H., Ayala-Orozco, B.: Lévy walk patterns in the foraging movements of spider monkeys (Ateles geoffroyi). Behavioral Ecology and Sociobiology 55(3), 223-230 (2004)

17. Reynolds, A.: How many animals really do the Lévy walk? Comment. Ecology 89(8), 2347-2351 (2008)

18. Reynolds, A.M.: Optimal random Lévy-loop searching: New insights into the searching behaviours of central-place foragers. EPL (Europhysics Letters) 82, 20001 (2008)

19. Reynolds, A.M., Frye, M.A.: Free-flight odor tracking in Drosophila is consistent with an optimal intermittent scale-free search. PLoS One 2(4), 354 (2007)

20. Shiner, J.S., Davison, M., Landsberg, P.T.: Simple measure for complexity. Physical Review E 59(2), 1459-1464 (1999)

21. Simoncelli, E., Adelson, E., Heeger, D.: Probability distributions of optical flow. In: Proceedings of IEEE Computer Society Conference on Computer Vision and Pattern Recognition, CVPR 1991, pp. 310-315. IEEE, Los Alamitos (2002)

22. Torralba, A.: Contextual priming for object detection. Int. J. of Comp. Vis. 53, 153-167 (2003)

23. Viswanathan, G.M., Afanasyev, V., Buldyrev, S.V., Murphy, E.J., Prince, P.A., Stanley, H.E.: Lévy flight search patterns of wandering albatrosses. Nature 381(6581), 413-415 (1996)

24. Viswanathan, G.M., Raposo, E.P., da Luz, M.: Lévy flights and superdiffusion in the context of biological encounters and random searches. Physics of Life Rev. 5(3), $133-150(2008)$ 